\title{
Almost Optimal Interior Penalty Discontinuous Approximations of Symmetric Elliptic Problems on Non- Matching Grids
}

R. D. Lazarov, J. E. Pasciak, J. Schöberl, and P. S.
Vassilevski

U.S. Department of Energy

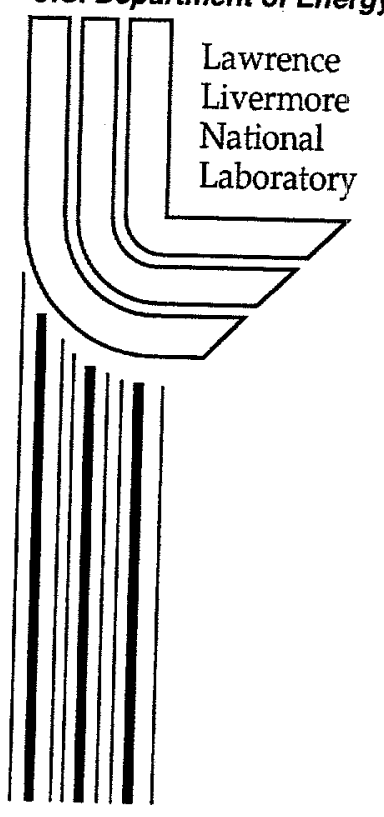

August 8, 2001 


\section{DISCLAIMER}

This document was prepared as an account of work sponsored by an agency of the United States Government. Neither the United States Government nor the University of California nor any of their employees, makes any warranty, express or implied, or assumes any legal liability or responsibility for the accuracy, completeness, or usefulness of any information, apparatus, product, or process disclosed, or represents that its use would not infringe privately owned rights. Reference herein to any specific commercial product, process, or service by trade name, trademark, manufacturer, or otherwise, does not necessarily constitute or imply its endorsement, recommendation, or favoring by the United States Government or the University of California. The views and opinions of authors expressed herein do not necessarily state or reflect those of the United States Government or the University of California, and shall not be used for advertising or product endorsement purposes.

This is a preprint of a paper intended for publication in a journal or proceedings. Since changes may be made before publication, this preprint is made available with the understanding that it will not be cited or reproduced without the permission of the author.

This work was performed under the auspices of the United States Department of Energy by the University of California, Lawrence Livermore National Laboratory under contract No. W-7405-Eng-48.

This report has been reproduced directly from the best available copy.

Available electronically at http://www.doc.gov/bridge

Available for a processing fee to U.S. Department of Energy

And its contractors in paper from

U.S. Department of Energy

Office of Scientific and Technical Information

P.O. Box 62

Oak Ridge, TN 37831-0062

Telephone: (865) 576-8401

Facsimile: (865) 576-5728

E-mail: reports@adonis.osti.gov

Available for the sale to the public from

U.S. Department of Commerce

National Technical Information Service

5285 Port Royal Road

Springfield, VA 22161

Telephone: (800) 553-6847

Facsimile: (703) 605-6900

E-mail: orders@ntis.fedworld.gov

Online ordering: http://www.ntis.gov/ordering.htm

OR

Lawrence Livermore National Laboratory

Technical Information Department's Digital Library

http://www.llnl.gov/tid/Library.html 


\title{
ALMOST OPTIMAL INTERIOR PENALTY DISCONTINUOUS APPROXIMATIONS OF SYMMETRIC ELLIPTIC PROBLEMS ON NON-MATCHING GRIDS
}

\author{
R.D. LAZAROV, J.E. PASCIAK, J. SCHÖBERL, AND P.S. VASSILEVSKI
}

\begin{abstract}
We consider an interior penalty discontinuous approximation for symmetric elliptic problems of second order on non-matching grids in this paer. The main result is an almost optimal error estimate for the interior penalty approximation of the original problem based on the partition of the domain into a finite number of subdomains. Further, an error analysis for the finite element approximation of the penalty formulation is given. Finally, numerical experiments on a series of model second order problems are presented.
\end{abstract}

\section{INTRODUCTION}

In this paper, we propose and analyze a simple strategy to construct composite discretizations of self-adjoint second order elliptic equations on non-matching grids. The need for discretizations on non-matching grids is motivated partially from the desire for parallel discretization methods (including adaptive) for PDEs, which is a much easier task if non-matching grids are allowed across the subdomain boundaries. Another situation may arise when different discretizations techniques are utilized in different parts of the subdomains and there is no a priori guarantee that the meshes will be aligned.

Our method can be described as interior penalty approximation based on partially discontinuous elements. The mortar method is a general technique for handling discretizations on non-matching grids. However, our motivation for using the penalty approach is that it eliminates the need for additional (Lagrange multiplier or mortar) spaces. There is a vast number of publications devoted to the mortar finite element method as a general strategy for deriving discretization methods on nonmatching grids. We refer the interested reader to the series of Proceedings of the International Conferences on Domain Decomposition Methods cf. e.g. [5], [11], [17] (for more information see, http://www.ddm.org).

In the present paper, we assume a model situation when the domain is split into a fixed number of non-overlapping subdomains and each subdomain is meshed

Date: August 31, 2000-beginning; Today is August 8, 2001.

1991 Mathematics Subject Classification. 65F10,65N20,65N30.

Key words and phrases. non-matching grids, interior penalty discretization, error estimates.

The work of the first and the second authors has been partially supported by the National Science Foundation under Grant DMS-9973328. The work of the last author was performed under the auspices of the U. S. Department of Energy by University of California Lawrence Livermore National Laboratory under contract W-7405-Eng-48. 
independently. This is a non-conforming method and the functions are discontinuous across the subdomain interfaces. The jump in the values of the functions along these interfaces is penalized in the variational formulation, a standard approach in the interior penalty method (cf. [2], [4], [14], [22]). For a recent comprehensive survey on this subject see [3]. An important feature of this approach is that we skip the term in the weak formulation that involves the co-normal derivative of the solution to the interface boundaries since the latter may lead to non-symmetric discretization (cf. [22]) of the original symmetric positive definite problem. An interior penalty finite element approximation with optimal condition number was proposed, studied, and tested on various examples in [20]. The error estimates derived in [20] were suboptimal with a loss of factor $h^{1 / 2-\delta}, 0<\delta<1 / 2$ for solutions in the Sobolev space $H^{2-\delta}(\Omega)$. In this paper we present a refined analysis and get almost optimal error estimates for linear finite element and solutions in $H^{2-\delta}(\Omega)$. In addition, we extend the analysis to decompositions with cross points. One can improve the accuracy somewhat for problems with smooth solutions by increasing the weight in the penalty term with the expense of increased condition number.

In the case of matching grids, finite element Galerkin method with penalty for a class of problems with discontinuous coefficients (interface problem) has been studied in [4]. Similarly, in [10], the interface problem has been addressed by recasting the problem as a system of first order (by introducing the gradient of the solution as a new vector variable) and applying the least-squares method for the system. Integrals of the squared jumps in the scalar and the normal component of the vector functions on the interface are added as penalty terms in the least-squares functional. In both cases an optimal with respect to the error method leads to a non-optimal condition number of the discrete problem.

Other approaches for handling discretizations on non-matching grids can involve different discretizations in the different subdomains. For example, mixed finite element method in one subdomain and standard Galerkin on the other (proposed in [25] and studied further in [18]), mixed finite element method and discontinuous Galerkin method cf. e.g., [13], or mixed finite element discretizations in both subdomains, cf. e.g., [1], [19]. Similarly, coupling finite volume and Galerkin methods has been proposed and studied in [15].

The structure of the present paper is as follows. In Section 2, we formulate the problem and its discretization. In Section 3, we introduce the primal and dual penalty formulations of the problem split into subproblems on nonoverlapping subdomains. In order to get an optimal estimate for the error in Section 4, we introduce the mixed formulation of the penalty problem and derive a fundamental a priori error estimate for its solution. In Section 5 , we analyse the difference between the solution of the original problem and the solution of the penalty formulation. The error is shown to be of almost optimal order for $u \in H^{2-\delta}(\Omega)$ for $\delta \geq 0$. For methods without cross-points the error is oprimal for $1 / 2>\delta>0$. Finally, the finite element discretization and its error analysis is presented in Section 6. Numerical test illustrating the accuracy of the method are given for two model problems. 


\section{NOTATIONS AND PROBLEM FORMULATION}

In this paper we use the standard notations for Sobolev spaces of functions defined in a bounded domain $\Omega \subset \mathcal{R}^{d}, d=2,3$. For example, $H^{s}(\Omega)$ for $s$ integer denotes the Hilbert space of functions $u$ defined on $\Omega$ and having generalized derivatives up to order $s$ that are square integrable in $\Omega$. For non-integer $s>0$ the spaces are obtained by the real method of interpolation (cf. [21]). $H_{0}^{1}(\Omega)$ is the space of functions in $H^{1}(\Omega)$ which vanish on $\partial \Omega$. The norm of $u \in H^{s}(\Omega)$ is denoted by $\|u\|_{s, \Omega}$. We also use the notation $|u|_{s, \Omega}$ for the $s$-order semi-norm in $H^{s}(\Omega)$. For the traces of functions in $H_{0}^{1}(\Omega)$ on a manifold $\Gamma$ of dimension $d-1$ (curves and surfaces) and $\partial \Gamma \subset \partial \Omega$, we will sometimes use the fractional order Sobolev spaces commonly denoted by $H_{00}^{1 / 2}(\Gamma)$ which is defined to be the interpolation space halfway between $H_{0}^{1}(\Gamma)$ and $L^{2}(\Gamma)$.

For a given Hilbert space $H$ with an inner product $(\cdot, \cdot)_{H}$ and corresponding norm $\|\cdot\|_{H}$ we denote by $H^{*}$ its dual, i.e. the space of all continuous linear functionals on $H$. We use the fact that $\left(H_{00}^{1 / 2}(\Gamma)\right)^{*}=H^{-1 / 2}(\Gamma)$.

For a given bounded polygon (polytope) $\Omega$, a source term $f \in L^{2}(\Omega)$, and coefficient matrix $a(x)$ that is symmetric and uniformly positive definite and bounded in $\Omega$, we consider the following model boundary value problem in a weak form: find $u \in H_{0}^{1}(\Omega)$ such that:

$$
A(u, v)=f(v) \quad \text { for all } v \in H_{0}^{1}(\Omega)
$$

Here

$$
A(u, v)=\int_{\Omega} a \nabla u \cdot \nabla v d x \text { and } f(v)=(f, v)_{0, \Omega}:=\int_{\Omega} f v d x
$$

\section{INTERIOR PENALTY FORMULATION}

We shall study a discretization of this problem by the finite element method using meshes that generally do not align along certain interfaces. This situation may arise when the domain $\Omega$ is split initially into a $p$ nonoverlapping subdomains $\Omega_{i}, i=1, \ldots, p$ and each subdomain is meshed (triangulated) independently of the others. We assume that the number of subdomains is fixed and each subdomain is a shape regular polyhedron. A model situation of this type for $d=2$ is shown on Figure 1. We denote by $\gamma_{i j}$ the interface between two subdomains $\Omega_{i}$ and $\Omega_{j}$ and by $\Gamma$ the union of all interfaces $\gamma_{i j}$. 


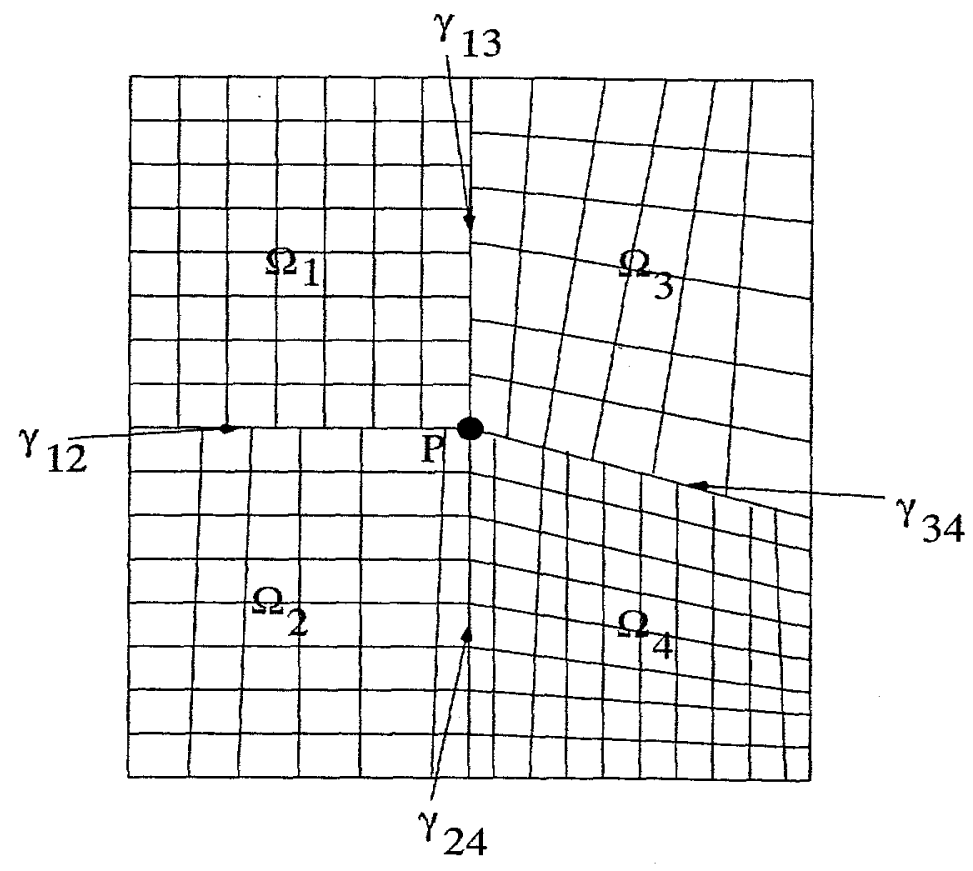

FIgurE 1. The domain $\Omega$ is partitioned into four subdomains $\Omega_{i}$ $i=1,2,3,4$ with interfaces $\gamma_{i j}$; each subdomain is partitioned into quadrilateral finite elements independently; $P$ is a cross point

We define

$$
\begin{aligned}
V & :=\left\{v \in L_{2}(\Omega):\left.v\right|_{\Omega_{i}} \in H^{1}\left(\Omega_{i}\right) \cap H_{0}^{1}(\Omega)\right\} \\
Q & :=L^{2}\left(\cup \partial \Omega_{i}\right) \\
a(u, v) & :=\sum(a \nabla u, \nabla v)_{0, \Omega_{i}}:=\sum a_{i}(u, v) \\
c(p, q) & :=(p, q)_{0, \Gamma}:=\int_{\Gamma} p q d s, \\
(p, q)_{0, \gamma_{i j}} & :=\int_{\gamma_{i j}} p q d s \\
\Lambda u & :=[u]
\end{aligned}
$$

Here the jump $[u]$ is defined as the difference of the traces of a function $u \in V$ on $\gamma_{i j}=\bar{\Omega}_{i} \cap \bar{\Omega}_{j}$. We specify a "master" side of each interface $\gamma_{i j}$ so on $\gamma_{i j}$ the jump is defined always as $[u]=\left.u\right|_{\Omega_{i}}-\left.u\right|_{\Omega_{j}}$, where $\Omega_{i}$ is the domain from the master side of $\gamma_{i j}$.

We approximate the original problem (2.1) by the following problem, which we further call the interior penalty formulation: Find $u_{\epsilon} \in V$ such that

$$
A^{\epsilon}\left(u_{\epsilon}, \varphi\right):=a\left(u_{\epsilon}, \varphi\right)+\epsilon^{-1} c\left(\Lambda u_{\epsilon}, \Lambda \varphi\right)=f(\varphi), \quad \text { for all } \varphi \in V .
$$


Here $\epsilon$ is a small parameter which later will be chosen as the mesh size of the finite element partition of $\Omega$. The problem (3.1) is further called the primal formulation to distinguish it from the the mixed formulation introduced in the next section.

The formulation (3.1) allows discontinuous solutions along the interface $\Gamma$. In order to control the size of the jump $\left[u_{\epsilon}\right]_{\Gamma}$ we have introduced it in the variational formulation as a least-squares penalty term with a large parameter $\epsilon^{-1}$. Our goal now is to estimate the difference $u-u_{\epsilon}$ assuming. that $u \in H^{2-\delta}(\Omega)$ with $0 \leq \delta<1 / 2$.

The bilinear form $A^{\epsilon}(\cdot, \cdot)$ defined in (3.1) is symmetric and positive definite. It is related to but much simpler than the corresponding discontinuous Galerkin method used in [2], [22]. The simplification comes from the fact that we do not have a term involving the co-normal derivative $a \nabla u \cdot \mathbf{n}$ along the interface $\Gamma$ with unit normal vector $\mathbf{n}$. This simplification comes at a cost: the proposed approximation will have almost optimal order of convergence for linear elements only, in contrast to the non-symmetric interior penalty Galerkin method studied in [22], where the optimal order is established for continuous finite elements of any degree. However, our formulation leads to a symmetric and positive definite problem which is more convenient for computational purposes.

\section{STUdY OF THE PRIMAL AND MIXED FORMULATIONS}

In this section, we shall study the solution of (3.1). This problem fits into the following general abstract class of parameter dependent problems. Let $\left(V,\|\cdot\|_{V},(\cdot, \cdot)_{V}\right)$ and $\left(Q,\|\cdot\|_{c}, c(\cdot, \circ)\right)$ be Hilbert spaces as illustrated earlier. Here the inner product $(\cdot, \cdot)_{V}$ defines a norm in $V$ and the norm $\|\cdot\|_{c}$ is defined by the inner product $c(\cdot, \cdot)$. We assume that we are given a continuous symmetric positive semi-definite bilinear form $a(\cdot, \cdot)$ on $V \times V$ and a continuous linear map $\Lambda: V \rightarrow Q$ so that

$$
a(v, w) \preceq\|v\|_{V}\|w\|_{V} \text { and }\|\Lambda v\|_{e} \preceq\|v\|_{V} \text {, for all } v, w \in V .
$$

Here and in the rest of the paper we use the signs $\preceq$ and $\succeq$ to denote inequalities with a constant that might depend on various parameters but is independent of $\epsilon$.

Next, we define

$$
A^{\epsilon}(v, w)=a(v, w)+\epsilon^{-1} c(\Lambda v, \Lambda w), \text { for all } v, w \in V
$$

We assume that the range of $\Lambda$ is dense in $Q$ but not necessarily closed. The parameter $\epsilon \in(0,1]$ is typically small. We further assume that $A^{1}(\cdot, \cdot)$ gives rise to an equivalent norm on $V$, i.e.,

$$
\|v\|_{V}^{2} \preceq A^{1}(v, v) \preceq\|v\|_{V}^{2}, \text { for all } v \in V .
$$

It easily follows that $A^{\epsilon}(\cdot, \cdot)$ is coercive on $V$ and satisfies

$$
\|v\|_{V}^{2} \preceq A^{\epsilon}(v, v) \preceq \epsilon^{-1}\|v\|_{V}^{2}, \text { for all } v \in V .
$$

Our approach is to reformulate (3.1) as a mixed problem as done in [24]. Let $u_{\epsilon}$ be the solution of (3.1) and define the dual variable $p_{\epsilon} \in Q$ by

$$
p_{\epsilon}:=\epsilon^{-1} \Lambda u_{\epsilon} .
$$


We get the mixed system for $u_{\epsilon}$ and $p_{\epsilon}$ :

$$
\begin{aligned}
a\left(u_{\epsilon}, v\right)+c\left(\Lambda v, p_{\epsilon}\right) & =f(v) \quad \text { for all } v \in V \\
c\left(\Lambda u_{\epsilon}, q\right)-\epsilon c\left(p_{\epsilon}, q\right) & =0 \quad \text { for all } q \in Q .
\end{aligned}
$$

Combining equations (4.3) and (4.4), and introducing the product space $X \equiv V \times Q$, we obtain the mixed variational problem: Find $u_{\epsilon} \in V$ and $p_{\epsilon} \in Q$ satisfying

$$
B^{\epsilon}\left(\left(u_{\epsilon}, p_{\epsilon}\right),(v, q)\right)=f(v) \quad \text { for all }(v, q) \in X,
$$

with the block bilinear form

$$
B^{\epsilon}\left(\left(u_{\epsilon}, p_{\epsilon}\right),(v, q)\right):=a\left(u_{\epsilon}, v\right)+c\left(\Lambda u_{\epsilon}, q\right)+c\left(\Lambda v, p_{\epsilon}\right)-\epsilon c\left(p_{\epsilon}, q\right) .
$$

The mixed bilinear form is well defined for the limit $\epsilon=0$. Any solution $\left(u_{\epsilon}, p_{\epsilon}\right)$ of (4.5) is in the space

$$
X_{0}=\{(v, q) \in X: \Lambda v=\epsilon q\} .
$$

This space will play an essential role in the analysis of the proposed interior penalty method. On the space $X \equiv V \times Q$, we define the norm

$$
\|(u, p)\|_{\epsilon}:=\left(\|u\|_{V}^{2}+\epsilon\|p\|_{c}^{2}\right)^{1 / 2}
$$

This norm degenerates to a semi-norm for $\epsilon=0$. The bilinear form $B^{\epsilon}(\cdot, \cdot)$ is continuous with parameter dependent bounds for that norm, namely, for $(u, p) \in X$ and $(v, q) \in X$

$$
\begin{aligned}
B^{\epsilon}((u, p),(v, q))= & a(u, v)+c(\Lambda u, q)+c(\Lambda v, p)-\epsilon c(p, q) \\
\preceq & \left(a(u, u)+\|\Lambda u\|_{c}^{2}+\|p\|_{c}^{2}+\epsilon\|p\|_{c}^{2}\right)^{1 / 2} \\
& \times\left(a(v, v)+\|\Lambda v\|_{c}^{2}+\|q\|_{c}^{2}+\epsilon\|q\|_{c}^{2}\right)^{1 / 2} \\
\preceq & \epsilon^{-1}\|(u, p)\| \epsilon\|(v, q)\|_{\epsilon} .
\end{aligned}
$$

On the other hand, $B^{\epsilon}(\cdot, \cdot)$ provides a uniformly continuous mapping from the dual of $X$ (with respect to $\|(\cdot, \cdot)\|_{\epsilon}$ ) into $X$. This is formulated in the following theorem:

Theorem 4.1. Let $f$ and $g$ be continuous linear functional on $V$ and $Q$ respectively. Then the extended mixed problem:

$$
B^{\epsilon}((u, p),(v, q))=f(v)+g(q) \quad \text { for all }(v, q) \in V \times Q
$$

has a unique solution $(u, p) \in X$. Moreover,

$$
\|u\|_{V}^{2}+\epsilon^{-1}\|\Lambda u\|_{c}^{2}+\epsilon\|p\|_{c}^{2} \preceq\|f\|_{V^{*}}^{2}+\epsilon^{-1}\|g\|_{Q^{*}}^{2} .
$$

Here $\|f\|_{V^{*}}$ and $\|g\|_{Q^{*}}$ denote the norms of the linear functionals.

Proof. First, we construct a solution by means of the primal problem. Since $\Lambda$ : $V \rightarrow Q$ is continuous, and $g(\cdot)$ is in $Q^{*}$, the functional $g(\Lambda \cdot)$ is continuous on $V$ :

$$
|g(\Lambda v)| \leq\|g\|_{Q^{*}}\|\Lambda v\|_{c} \preceq\|g\|_{Q^{*}}\|v\|_{V} .
$$

Let $u \in V$ be the solution of

$$
a(u, v)+\epsilon^{-1} c(\Lambda u, \Lambda v)=f(v)+\epsilon^{-1} g(\Lambda v) \quad \text { for all } v \in V .
$$


We use the ellipticity $(4.1)$ of $a(\cdot, \cdot)+c(\Lambda \cdot, \Lambda \cdot)$ to get

$$
\begin{aligned}
\|u\|_{V}^{2}+\epsilon^{-1}\|\Lambda u\|_{c}^{2} & \preceq a(u, u)+\epsilon^{-1} c(\Lambda u, \Lambda u) \\
& =f(u)+\epsilon^{-1} g(\Lambda u) \\
& \preceq\|f\|_{V^{*}}\|u\|_{V}+\epsilon^{-1 / 2}\|g\|_{Q^{*}} \epsilon^{-1 / 2}\|\Lambda u\|_{c} \\
& \preceq\left(\|f\|_{V^{*}}^{2}+\epsilon^{-1}\|g\|_{Q^{*}}^{2}\right)^{1 / 2}\left(\|u\|_{V}^{2}+\epsilon^{-1}\|\Lambda u\|_{c}^{2}\right)^{1 / 2} .
\end{aligned}
$$

Dividing by $\left(\|u\|_{V}^{2}+\epsilon^{-1}\|\Lambda u\|_{c}^{2}\right)^{1 / 2}$ gives the bound for $u$. By the Riesz Representation Theorem we define $\tilde{g} \in Q$ such that

$$
c(\tilde{g}, q)=g(q) \quad \text { for all } q \in Q
$$

and find that

$$
p=\epsilon^{-1}(\Lambda u-\tilde{g})
$$

Clearly,

$$
\epsilon\|p\|_{c}^{2} \preceq \epsilon^{-1}\|\Lambda u\|_{c}^{2}+\epsilon^{-1}\|g\|_{Q^{*}}^{2} \preceq\|f\|_{V^{*}}^{2}+\epsilon^{-1}\|g\|_{Q^{*}}^{2} .
$$

We verify that $(u, p)$ is a solution of (4.10). Indeed, for all $(v, q) \in X$,

$$
\begin{aligned}
B^{\epsilon}((u, p),(v, q)) & =a(u, v)+c(\Lambda u, q)+c\left(\Lambda v, \epsilon^{-1}(\Lambda u-\tilde{g})\right)-\epsilon c\left(\epsilon^{-1}(\Lambda u-\tilde{g}), q\right) \\
& =a(u, v)+\epsilon^{-1} c(\Lambda u, \Lambda v)-\epsilon^{-1} c(\tilde{g}, \Lambda v)+c(\tilde{g}, q) \\
& =f(v)+g(q) .
\end{aligned}
$$

Finally, we prove that the solution is unique. Any solution $(u, p)$ of the homogenous problem satisfies

$$
\begin{aligned}
0 & =B^{\epsilon}((u, p),(u, \Lambda u-p)) \\
& =a(u, u)+c(\Lambda u, \Lambda u)+\epsilon c(p, p)-\epsilon c(p, \Lambda u) \\
& \geq a(u, u)+\left(1-\frac{\epsilon}{2}\right) c(\Lambda u, \Lambda u)+\frac{\epsilon}{2} c(p, p) .
\end{aligned}
$$

Thus, zero is the only solution of the homogeneous equation and the proof is complete.

We will now demonstrate the gain in using the mixed form. Namely, in Theorem 4.3 we will show a uniform in $\epsilon>0$ a priori estimate of the solution to the problem (4.10).

Let us define the norm $\|p\|_{Q, 0}$ for $p \in Q$ by

$$
\|p\|_{Q, 0}=\sup _{v \in V} \frac{c(p, \Lambda v)}{\|v\|_{V}}
$$

That this is a norm follows from the assumption that $\Lambda V$ is dense in $Q$. Let $Q_{0}$ denote the closure of $\Lambda V$ in the norm $\|\cdot\|_{Q, 0}$. In general, $\|\cdot\|_{Q, 0}$ is a weaker norm than $\|.\|_{c}$. By definition, $\Lambda$ has a closed range in $Q_{0}^{*}$. In the limit case $\epsilon=0$ the bilinear form $B^{\epsilon}((u, p),(v, q))$ is continuous and stable on $V \times Q_{0}$ : 
Theorem 4.2 (Brezzi, see, e.g. [9], Proposition 1.3). The bilinear form

$$
B^{0}((u, p),(v, q))=a(u, v)+c(\Lambda u, q)+c(\Lambda v, p)
$$

is continuous, i.e.

$$
B^{0}((u, p),(v, q)) \preceq\left(\|u\|_{V}^{2}+\|p\|_{Q, 0}^{2}\right)^{1 / 2}\left(\|v\|_{V}^{2}+\|q\|_{Q, 0}^{2}\right)^{1 / 2}
$$

and stable, i.e.

$$
\sup _{u \in V, p \in Q_{0}} \frac{B^{0}((u, p),(v, q))}{\left(\|u\|_{V}^{2}+\|p\|_{Q, 0}^{2}\right)^{1 / 2}} \succeq\left(\|v\|_{V}^{2}+\|q\|_{Q, 0}^{2}\right)^{1 / 2}
$$

on the space $V \times Q_{0}$.

For the case $\epsilon>0$, we need a norm depending on the parameter $\epsilon$. We define

$$
\|p\|_{Q}:=\|p\|_{Q, \epsilon}:=\left(\|p\|_{Q, 0}^{2}+\epsilon\|p\|_{c}^{2}\right)^{1 / 2} \text {. }
$$

This norm is equivalent to $\|.\|_{c}$ for fixed $\epsilon>0$, but not necessarily uniformly equivalent with respect to $\epsilon$ since obviously $\epsilon\|p\|_{c}^{2} \leq \| p_{Q}^{2}$. We define the product space

$$
\mathcal{X}=V \times Q
$$

with the norm

$$
\|(u, p)\|_{\mathcal{X}}=\left(\|u\|_{V}^{2}+\|p\|_{Q}^{2}\right)^{1 / 2}
$$

The following theorem states that $B^{\epsilon}(\cdot, \cdot)$ is bounded in $\mathcal{X}$ and satisfies an inf-sup condition with a constant independent of $\epsilon$ :

Theorem 4.3. Assume that (4.1) is satisfied. Let $B^{\epsilon}(\cdot, \cdot)$ and $\|\cdot\| x$ be defined by (4.5) and (4.16), respectively. Then:

- The bilinear form $B^{\epsilon}(\cdot, \cdot)$ is uniformly continuous on $\mathcal{X}$, i.e.

$$
B^{\epsilon}((u, p),(v, q)) \preceq\|(u, p)\|_{\mathcal{X}}\|(v, q)\|_{\mathcal{X}} \quad \text { for all }(u, p),(v, q) \in \mathcal{X} ;
$$

- The bilinear form $B^{\epsilon}(\cdot, \cdot)$ is uniformly stable on $\mathcal{X}$, i.e.

$$
\sup _{(u, p) \in \mathcal{X}} \frac{B^{\varepsilon}((u, p),(v, q))}{\|(u, p)\| \mathcal{X}} \succeq\|(v, q)\|_{\mathcal{X}} \quad \text { for all }(v, q) \in \mathcal{X}
$$

- the mixed problem $B^{\epsilon}((u, p),(v, q))=f(v)+g(q)$ for all $(v, q) \in V \times Q$ has unique solution for any $f \in V^{*}$ and $g \in Q^{*}$ and the solution satisfies the a priori estimate:

$$
\|(u, p)\|_{\mathcal{X}} \preceq\|f\|_{V^{*}}+\|g\|_{Q^{*}}
$$

Proof. The proof of the continuity follows estimate (4.9) but due to the new stronger norm in $Q$ (see, (4.15)) we have an improved estimate for the mixed term:

$$
c(\Lambda u, q) \leq\|u\|_{V} \sup _{v \in V} \frac{c(\Lambda v, q)}{\|v\|_{V}}=\|u\|_{V}\|q\|_{Q, 0}
$$

Thus we get uniform continuity. 
We need only verify (4.18). To this end, fix $(v, q) \in \mathcal{X}$. By definition of the norm $\|.\|_{Q, 0}$, there exists a $\tilde{v} \in V$ such that

$$
\frac{c(\Lambda \tilde{v}, q)}{\|\tilde{v}\|_{V}} \succeq\|q\|_{Q, 0}
$$

We are free to scale $\tilde{v}$ such that

$$
\|\tilde{v}\|_{V}=\|q\|_{Q, 0} \quad \text { and } \quad c(\Lambda \tilde{v}, q) \succeq\|q\|_{Q, 0}^{2} .
$$

Let $(\tilde{u}, \tilde{p})$ be the unique solution (by Theorem 4.1) of

$$
B^{\epsilon}((\tilde{u}, \tilde{p}),(w, r))=(v, w)_{V}+c(\Lambda \tilde{v}, r)+\epsilon c(q, r) \quad \text { for all }(w, r) \in \mathcal{X} .
$$

We will use $(\tilde{u}, \tilde{p})$ in order to verify (4.18). First, we see that

$$
\begin{aligned}
B^{\epsilon}((\tilde{u}, \tilde{p}),(v, q)) & =(v, v)_{V}+c(\Lambda \tilde{v}, q)+\epsilon c(q, q) \\
& \succeq\|v\|_{V}^{2}+\|q\|_{Q, 0}^{2}+\epsilon\|q\|_{c}^{2} \\
& =\|(v, q)\|_{\mathcal{X}}^{2},
\end{aligned}
$$

so that

$$
\sup _{(u, p) \in \mathcal{X}} \frac{B^{\epsilon}((u, p),(v, q))}{\|(u, p)\|_{\mathcal{X}}} \geq \frac{B^{\epsilon}((\tilde{u}, \tilde{p}),(v, q))}{\|(\tilde{u}, \tilde{p})\|_{\mathcal{X}}} \succeq \frac{\|(v, q)\|_{\mathcal{X}}^{2}}{\|(\tilde{u}, \tilde{p})\|_{\mathcal{X}}} .
$$

Thus, we need only to show that

$$
\|(\tilde{u}, \tilde{p})\|_{\mathcal{X}} \preceq\|(v, q)\|_{\mathcal{X}} .
$$

By the definition of $B^{\epsilon}(\cdot, \cdot)$ and $(4.20)$, for all $(w, r) \in \mathcal{X}$,

$$
\begin{aligned}
B^{\epsilon}((\tilde{u}-\tilde{v}, \tilde{p}),(w, r)) & =B^{\epsilon}((\tilde{u}, \tilde{p}),(w, r))-B^{\epsilon}((\tilde{v}, 0),(w, r)) \\
& =(v, w)_{V}+c(\Lambda \tilde{v}, r)+\epsilon c(q, r)-[a(\tilde{v}, w)+c(\Lambda \tilde{v}, r)] \\
& =(v, w)_{V}-a(\tilde{v}, w)+\epsilon c(q, r) .
\end{aligned}
$$

Applying Theorem 4.1 gives

$$
\|\tilde{u}-\tilde{v}\|_{V}^{2}+\epsilon\|\tilde{p}\|_{c}^{2} \preceq\|v\|_{V}^{2}+\|\tilde{v}\|_{V}^{2}+\epsilon\|q\|_{c}^{2} .
$$

Thus,

$$
\begin{aligned}
\|\tilde{u}\|_{V}^{2}+\epsilon\|\tilde{p}\|_{c}^{2} & \preceq\|v\|_{V}^{2}+\|q\|_{Q, 0}^{2}+\epsilon\|q\|_{c}^{2} \\
& =\|(v, q)\|_{X}^{2} .
\end{aligned}
$$

Finally, we need to estimate $\|\tilde{p}\|_{Q, 0}$. Using $(w, 0)$ in $(4.20)$ gives

$$
B^{\epsilon}((\tilde{u}, \tilde{p}),(w, 0)) \equiv a(\tilde{u}, w)+c(\Lambda w, \tilde{p})=(w, v)_{V} \text {, for all } w \in V .
$$

Consequently,

$$
\begin{aligned}
\|\tilde{p}\|_{Q, 0} & =\sup _{w \in V} \frac{c(\Lambda w, \tilde{p})}{\|w\|_{V}}=\sup _{w \in V} \frac{(w, v)_{V}-a(\tilde{u}, w)}{\|w\|_{V}} \\
& \preceq\|v\|_{V}+\|\tilde{u}\|_{V} \preceq\|(v, q)\|_{\mathcal{X}} .
\end{aligned}
$$


Combining the above estimates completes the proof.

\section{ANALYSIS OF THE INTERIOR PENALTY APPROXIMATION}

In this section, we derive the basic error estimates for the proposed interior penalty method (3.1). We present the estimate for the general case when the partition of $\Omega$ into subdomains $\Omega_{i}$ has "cross-points" (see, Figure 1). For $d=2$ the cross-points are the end points of the edges $\gamma_{i j}$ that are in the interior of $\Omega$. For $d=3$ the cross-points are the edges of $\gamma_{i j}$ that are in the interior of $\Omega$. The case of absence of cross-point is somewhat simpler and is discussed at the end of this section.

Here we use some fundamental results from the domain decomposition literature (see, e.g. $[6,7]$ ). Since all subdomains $\Omega_{i}$ are shape regular the estimates

$$
\left\|\left.v\right|_{\gamma_{i j}}\right\|_{H_{00}^{1 / 2}\left(\gamma_{i j}\right)} \preceq\|v\|_{H^{1}\left(\Omega_{i}\right)}
$$

hold for functions $v \in H^{1}\left(\Omega_{i}\right)$ which vanish on $\partial \Omega_{i} \backslash \gamma_{i j}$. Here $\left.v\right|_{\gamma_{i j}}$ is the trace of $v$ on $\gamma_{i j}$. We note also that given any $\sigma_{i j} \in H_{00}^{1 / 2}\left(\gamma_{i j}\right)$, there is an extension $v$ satisfying the above estimates. The following proposition plays a key role in the proof of the error estimate for the interior penalty method.

Proposition 5.1. For any $\epsilon>0$ and $\lambda \in L^{2}(\Gamma),\left.\lambda\right|_{\gamma_{i j}} \in H^{1 / 2}\left(\gamma_{i j}\right)$ the following estimate is valid:

$$
\|\lambda\|_{Q^{*}} \leq c \log \epsilon^{-1}\left(\sum_{\gamma_{i j}}\|\lambda\|_{H^{1 / 2}\left(\gamma_{i j}\right)}^{2}\right)^{1 / 2} .
$$

The constant $c$ is independent of $\epsilon$ but depends on the shape and the number of subdomains.

The proof of this estimate is given at the end of this section. We now prove the main result in this section:

Theorem 5.1. Assume that the solution $u$ of (2.1) is in $H^{2-\delta}(\Omega)$ for some $\delta \in$ $[0,1 / 2)$. Then

$$
\left\|u-u_{\epsilon}\right\|_{V}+\left\|p-p_{\epsilon}\right\|_{Q} \leq c \epsilon^{1-\delta}\left(\log \epsilon^{-1}\right)^{1-2 \delta}|| u \|_{H^{2-\delta}(\Omega)}, \quad 0 \leq \delta \leq 1 / 2 .
$$

Here the norm in $Q$ is defined as in (4.15) and the constant $c$ is independent of $\epsilon$.

Proof. We first note that the solution $u$ of the problem (2.1) satisfies the identity,

$$
A^{\epsilon}(u, \varphi)=f(\varphi)+c(a \nabla u \cdot \mathbf{n}, \Lambda \varphi), \quad \text { for all } \varphi \in V,
$$

where the normal vector $\mathbf{n}$ is always pointing outward from the master side of $\gamma_{i j}$. Here we have used the fact that the exact solution has continuous normal flux, i.e., in particular, $\left.[a \nabla u \cdot \mathbf{n}]\right|_{\Gamma}=0$. To simplify the notations, we define the function

$$
\theta=a \nabla u \cdot \mathbf{n} \text { on } \Gamma \text {. }
$$

Further, the penalty solution $u_{\epsilon}$ has been defined as a solution to $A^{\epsilon}\left(u_{\epsilon}, \varphi\right)=f(\varphi)$. Subtracting these two identities we get the following equation for the error $e=u-u_{\epsilon}$ :

$$
A^{\epsilon}(e, \varphi)=c(\theta, \Lambda \varphi) \text { for all } \varphi \in V \text {. }
$$


In order to use the a priori estimates of the mixed setting we shall put this problem again in a mixed form. Namely, we introduce a new dependent variable $E:=a \nabla u \cdot \mathbf{n}-\epsilon^{-1} \Lambda e:=\theta-\epsilon^{-1} \Lambda e$ defined on $\Gamma$ so that the pair $(e, E)$ satisfies:

$$
B^{\epsilon}((e, E),(v, q))=\epsilon c(\theta, q) \quad \text { for all }(v, q) \in V \times Q \text {. }
$$

The estimate (4.19) will provide a basis for the analysis of the error $(e, E)$, namely,

$$
\|e\|_{V}+\|E\|_{Q} \preceq \epsilon \sup _{q \in Q} \frac{c(\theta, q)}{\|q\|_{Q}} .
$$

Since $\|q\|_{Q} \geq \epsilon^{1 / 2}\|q\|_{c}$ we easily get

$$
\|e\|_{V}+\|E\|_{Q} \preceq \sqrt{\epsilon}\|\theta\|_{0, \Gamma} .
$$

This estimate is an easy corollary of the set up of the problem but it yields an error for the interior penalty method of order at most $O\left(\epsilon^{1 / 2}\right)$. We can improve it when $\theta$ is a smoother function. To accomplish this we first apply estimate (5.2) for $\lambda=\theta$ to get get:

$$
\|e\|_{V}+\|E\|_{Q} \preceq \epsilon\|\theta\|_{Q^{*}} \preceq \epsilon \log \epsilon^{-1}\left(\sum_{\gamma_{i j}}\|\theta\|_{H^{1 / 2}\left(\gamma_{i j}\right)}^{2}\right)^{1 / 2} .
$$

Second, we use the interpolation space $H^{1 / 2-\delta}\left(\gamma_{i j}\right), 0 \leq \delta \leq 1 / 2$, between the spaces $L^{2}\left(\gamma_{i j}\right)$ and $H^{1 / 2}\left(\gamma_{i j}\right)$ so that

$$
\|\theta\|_{H^{1 / 2-\delta}\left(\gamma_{i j}\right)} \preceq\|\theta\|_{H^{1 / 2}\left(\gamma_{i j}\right)}^{1-2 \delta}\|\theta\|_{0, \gamma_{i j}}^{2 \delta} .
$$

Next, we observe that interpolated norm with $\delta \in[0,1 / 2]$ between

$$
\left(\sum_{\gamma_{i j}}\|\theta\|_{0, \gamma_{i j}}^{2}\right)^{1 / 2} \text { and }\left(\sum_{\gamma_{i j}}\|\theta\|_{H^{1 / 2}\left(\gamma_{i j}\right)}^{2}\right)^{1 / 2} \text { is bounded by }\left(\sum_{\gamma_{i j}}\|\theta\|_{H^{1 / 2-\delta}\left(\gamma_{i j}\right)}^{2}\right)^{1 / 2} \text {. }
$$

This fact follows from the definition of the real interpolation method [21].

Finally, for $u \in H^{2-\delta}(\Omega), 0 \leq \delta \leq 1 / 2$, one can show that

$$
\|\theta\|_{H^{1 / 2-\delta}\left(\gamma_{i j}\right)}=\|a \nabla u \cdot \mathbf{n}\|_{H^{1 / 2-\delta}\left(\gamma_{i j}\right)} \preceq\|u\|_{H^{2-\delta}\left(\Omega_{i}\right)} .
$$

Interpolating estimates (5.5) (5.6) gives the desired results (5.3). This completes the proof of the theorem.

In the rest of this section, we give a proof of Proposition 5.1. This follows immediately from the three lemmas below. The first lemma follows easily from the extension noted at the beginning of this section.

Lemma 5.1. For any given $\sigma_{i j} \in H_{00}^{1 / 2}\left(\gamma_{i j}\right)$ on all $\gamma_{i j} \subset \Gamma$ there exists a $v \in V$ such that

$$
[v]_{\gamma_{i j}}=\sigma_{i j}
$$

and

$$
\|v\|_{V} \preceq\left(\sum\left\|\sigma_{i j}\right\|_{H_{00}^{1 / 2}\left(\gamma_{i j}\right)}^{2}\right)^{1 / 2} .
$$


Next, for $\mu \in L^{2}\left(\gamma_{i j}\right)$ we define the norm

$$
\|\mu\|_{Q_{i j}}:=\left(\|\mu\|_{H^{-1 / 2}\left(\gamma_{i j}\right)}^{2}+\epsilon\|\mu\|_{0, \gamma_{i j}}^{2}\right)^{1 / 2}
$$

and its dual

$$
\|\mu\|_{Q_{i j}^{*}}:=\sup _{\lambda \in L^{2}\left(\gamma_{i j}\right)} \frac{(\lambda, \mu)_{0, \gamma_{i j}}}{\|\lambda\|_{Q_{i j}}}
$$

Note that the space $Q$ and its dual have been defined in Section 4 . We then have the following lemma.

Lemma 5.2. For all $\lambda \in Q^{*}$,

$$
\|\lambda\|_{Q^{*}} \preceq\left(\sum_{\gamma_{i j}}\|\lambda\|_{Q_{Q_{j}^{*}}^{*}}^{2}\right)^{1 / 2} .
$$

Proof. Let $\mu \in Q$ be non-zero. First, we verify that

$$
\sum_{\gamma_{i j}} \sup _{\sigma_{i j} \in H_{00}^{1 / 2}\left(\gamma_{i j}\right)} \frac{\left(\mu, \sigma_{i j}\right)_{0, \gamma_{i j}}^{2}}{\left\|\sigma_{i j}\right\|_{H_{00}^{1 / 2}\left(\gamma_{i j}\right)}^{2}} \preceq \sup _{v \in V} \frac{(\mu,[v])_{0, \Gamma}^{2}}{\|v\|_{V}^{2}}
$$

Set $\bar{\sigma}_{i j}=\alpha \sigma_{i j}$ where $\alpha$ is chosen such that $\left\|\bar{\sigma}_{i j}\right\|_{H_{00}^{1 / 2}\left(\gamma_{i j}\right)}^{2}=\left(\mu, \bar{\sigma}_{i j}\right)_{0, \gamma_{i j}}$. By Lemma 5.1, there exists an extension $v \in V$ such that

$$
[v]_{\gamma_{i j}}=\bar{\sigma}_{i j} \quad \text { and } \quad\|v\|_{V}^{2} \preceq \sum_{\bar{\gamma}_{i j}}\left\|\bar{\sigma}_{i j}\right\|_{H_{00}^{1 / 2}\left(\gamma_{i j}\right)}^{2} .
$$

Then,

$$
\begin{aligned}
\sum_{\gamma_{i j}} \frac{\left(\mu, \sigma_{i j}\right)_{0, \gamma_{i j}}^{2}}{\left\|\sigma_{i j}\right\|_{H_{00}^{1 / 2}\left(\gamma_{i j}\right)}^{2}} & =\sum_{\gamma_{i j}}\left(\mu, \bar{\sigma}_{i j}\right)_{0, \gamma_{i j}}=(\mu,[v])_{0, \Gamma}=\frac{(\mu,[v])_{0, \Gamma}^{2}}{\|v\|_{V}^{2}} \frac{\|v\|_{V}^{2}}{(\mu,[v])_{0, \Gamma}} \\
& \preceq \frac{(\mu,[v])_{0, \Gamma}^{2}}{\|v\|_{V}^{2}} \frac{\sum_{\gamma_{i j}}\left\|\bar{\sigma}_{i j}\right\|_{H_{00}^{1 / 2}\left(\gamma_{i j}\right)}^{2}}{\sum_{\gamma_{i j}}\left(\mu, \bar{\sigma}_{i j}\right)_{0, \gamma_{i j}}}=\frac{(\mu,[v])_{0, \Gamma}^{2}}{\|v\|_{V}^{2}} .
\end{aligned}
$$

The inequality (5.8) follows.

It immediately follows from (5.8) that

$$
\sum_{\gamma_{i j}}\|\mu\|_{Q_{i j}}^{2} \preceq\|\mu\|_{Q}^{2}
$$


We continue with

$$
\begin{aligned}
(\lambda, \mu)_{0, \Gamma} & =\sum_{\gamma_{i j}}(\lambda, \mu)_{0, \gamma_{i j}} \leq \sum_{\gamma_{i j}}\|\lambda\|_{Q_{i j}^{*}}\|\mu\|_{Q_{i j}} \\
& \leq\left(\sum_{\gamma_{i j}}\|\lambda\|_{Q_{i j}^{*}}^{2}\right)^{1 / 2}\left(\sum_{\gamma_{i j}}\|\mu\|_{Q_{i j}}^{2}\right)^{1 / 2} \\
& \preceq\left(\sum_{\gamma_{i j}}\|\lambda\|_{Q_{i j}^{*}}^{2}\right)^{1 / 2}\|\mu\|_{Q} .
\end{aligned}
$$

The lemma follows dividing by $\|\mu\|_{Q}$ and taking the supremum.

Lemma 5.3. For $\lambda \in L^{2}\left(\gamma_{i j}\right)$,

$$
\|\lambda\|_{Q_{i j}^{*}} \preceq \log \epsilon^{-1}\|\lambda\|_{H^{1 / 2}\left(\gamma_{i j}\right)} .
$$

Proof. The proof of this lemma is based on techniques from the analysis of domain decomposition preconditioners. We illustrate the proof in the case of three spatial dimensions. The two dimensional case is similar.

Let $\lambda$ be in $L^{2}\left(\gamma_{i j}\right)$ and $S_{\epsilon}$ be a finite element sub-space of $H^{1}\left(\gamma_{i j}\right)$ of quasiuniform mesh-size $\epsilon$. The $L^{2}$-orthogonal projection operator $\mathcal{Q}$ onto $S_{\epsilon}$ is bounded on $H^{1 / 2}\left(\gamma_{i j}\right)$ and satisfies

$$
\epsilon^{-1 / 2}\|\lambda-\mathcal{Q} \lambda\|_{0, \gamma_{i j}}+\|\mathcal{Q} \lambda\|_{H^{1 / 2}\left(\gamma_{i j}\right)} \leq c\|\lambda\|_{H^{1 / 2}\left(\gamma_{i j}\right)}
$$

We first split $\lambda=(\lambda-\mathcal{Q} \lambda)+\mathcal{Q} \lambda$, and further decompose the finite element part

$$
\mathcal{Q} \lambda=\lambda_{1}+\lambda_{2}
$$

such that $\lambda_{1}=\mathcal{Q} \lambda$ on $\partial \gamma_{i j}$ and $\lambda_{1}=0$ on all interior nodes of $\gamma_{i j}$ ( $\lambda_{2}$ being the remainder vanishing at $\partial \gamma_{i j}$ ).

A simple transformation argument and Lemma 4.2 of [7] gives

$$
\left\|\lambda_{1}\right\|_{0, \gamma_{i j}} \preceq \epsilon^{1 / 2}\left\|\lambda_{1}\right\|_{L^{2}\left(\partial \gamma_{i j}\right)} \preceq \epsilon^{1 / 2}\left(\log \epsilon^{-1}\right)^{1 / 2}\|\mathcal{Q} \lambda\|_{H^{1 / 2}\left(\gamma_{i j}\right)} .
$$

Lemma 4.3 of $[7]$ gives

$$
\left\|\lambda_{2}\right\|_{H_{00}^{1 / 2}\left(\gamma_{i j}\right)} \preceq \log \epsilon^{-1}\|\mathcal{Q} \lambda\|_{H^{1 / 2}\left(\gamma_{i j}\right)} .
$$

Now we use the above splitting to get

$$
\|\lambda\|_{Q_{i j}^{*}}=\sup _{\mu \in L^{2}\left(\gamma_{i j}\right)} \frac{(\lambda, \mu)_{0, \gamma_{i j}}}{\|\mu\|_{Q_{i j}}}=\sup _{\mu \in L^{2}\left(\gamma_{i j}\right)} \frac{(\lambda-\mathcal{Q} \lambda, \mu)_{0, \gamma_{i j}}+\left(\lambda_{1}, \mu\right)_{0, \gamma_{i j}}+\left(\lambda_{2}, \mu\right)_{0, \gamma_{i j}}}{\|\mu\|_{H^{-1 / 2}\left(\gamma_{i j}\right)}+\epsilon^{1 / 2}\|\mu\|_{0, \gamma_{i j}}} .
$$

Further, using the estimate (5.11) we have

$$
\begin{aligned}
\sup _{\mu \in L^{2}\left(\gamma_{i j}\right)} \frac{(\lambda-\mathcal{Q} \lambda, \mu)_{0, \gamma_{i j}}}{\|\mu\|_{H^{-1 / 2}\left(\gamma_{i j}\right)}+\epsilon^{1 / 2}\|\mu\|_{0, \gamma_{i j}}} & \leq \sup _{\mu \in L^{2}\left(\gamma_{i j}\right)} \frac{\|\lambda-\mathcal{Q} \lambda\|_{0, \gamma_{i j}}\|\mu\|_{0, \gamma_{i j}}}{\|\mu\|_{H^{-1 / 2}\left(\gamma_{i j}\right)}+\epsilon^{1 / 2}\|\mu\|_{0, \gamma_{i j}}} \\
& \preceq\|\lambda\|_{H^{1 / 2}\left(\gamma_{i j}\right)} .
\end{aligned}
$$

Similarly, using the estimates for $\lambda_{1}$ and $\lambda_{2}$ we get

$$
\left(\lambda_{1}, \mu\right)_{0, \gamma_{i j}} \leq\left\|\lambda_{1}\right\|_{0, \gamma_{i j}}\|\mu\|_{0, \gamma_{i j}} \preceq \epsilon^{1 / 2}\left(\log \epsilon^{-1}\right)^{1 / 2}\|\lambda\|_{H^{1 / 2}\left(\gamma_{i j}\right)}\|\mu\|_{0, \gamma_{i j}}
$$


and

$$
\left(\lambda_{2}, \mu\right)_{0, \gamma_{i j}} \leq\left\|\lambda_{2}\right\|_{H_{00}^{1 / 2}\left(\gamma_{i j}\right)}\|\mu\|_{H^{-1 / 2}\left(\gamma_{i j}\right)} \preceq \log \epsilon^{-1}\|\mathcal{Q} \lambda\|_{H^{1 / 2}\left(\gamma_{i j}\right)}\|\mu\|_{H^{-1 / 2}\left(\gamma_{i j}\right)}
$$

Finally, combining the estimates for all three parts, we complete the proof:

$$
\|\lambda\|_{Q_{i j}^{*}}=\sup _{\mu \in L^{2}\left(\gamma_{i j}\right)} \frac{(\lambda, \mu)_{0, \gamma_{i j}}}{\|\mu\|_{Q_{i j}}} \preceq \log \epsilon^{-1}\|\lambda\|_{H^{1 / 2}\left(\gamma_{i j}\right)} .
$$

In the case of no cross points, we can get a slightly better result. In this case $\gamma_{i j}=\Gamma$ and the assumption stated at the beginning of this section holds. The following theorem provides an error estimate in this case.

Theorem 5.2. In the case of absence of "cross-points" the following estimate holds

$$
\|e\|_{V}+\|E\|_{Q} \preceq \epsilon^{1-\delta}\|u\|_{H^{2-\delta}(\Omega)} .
$$

for $u \in H^{2-\delta}(\Omega), 0 \leq \delta<1 / 2$.

Proof. Since there are no "cross-points" for $v \in V$ the jump $[v]=\Lambda v$ is in $H_{00}^{1 / 2}(\Gamma)$. Therefore, there is an extension, which satisfies (5.1) so that

$$
\|q\|_{Q} \succeq \sup _{v \in V} \frac{c(\Lambda v, q)}{\|v\|_{V}} \succeq\|q\|_{H^{-1 / 2}(\Gamma)}
$$

This implies

$$
\sup _{q \in Q} \frac{c(\theta, q)}{\|q\|_{Q}} \preceq \sup _{q \in Q} \frac{c(\theta, q)}{\|q\|_{H^{-1 / 2}(\Gamma)}} \preceq\|\theta\|_{H_{00}^{1 / 2}(\Gamma)}
$$

so that

$$
\|e\|_{V}+\|E\|_{Q} \preceq \epsilon\|\theta\|_{H_{00}^{1 / 2}(\Gamma)} .
$$

Interpolating (5.5) and (5.13) we get

$$
\|e\|_{V}+\|E\|_{Q} \preceq \epsilon^{1-\delta}\|\theta\|_{H^{1 / 2-\delta}(\Gamma)} .
$$

The result then follows from the trace estimate

$$
\|\theta\|_{H^{1 / 2-\delta}(\Gamma)} \preceq\|u\|_{H^{2-\delta}(\Omega)}
$$

which holds for polygonal interface $\Gamma$ (cf. [16]).

\section{Finite element aPPROXimation of the PENALTy FORMULATION}

6.1. Finite element formulation and error analysis. Now we disretize the problem (3.1) by the finite element method. Each subdomain $\Omega_{i}$ is meshed independently by a quasi-uniform and shape-regular triangulation $\mathcal{T}_{i}$ and consequently the whole domain has a finite element splitting $\mathcal{T}=\cup_{i} \mathcal{T}_{i}$. Quasi-uniformity of the mesh means that for $\tau \in \mathcal{T}$ and $h_{\tau}=\operatorname{diam}(\tau),|\tau|=\operatorname{meas}(\tau)$ we have $|\tau| \approx h_{\tau}^{d}$, where $d=2,3$ is the dimension of the space. We shall use also the global mesh-size parameter

$$
h=\max _{\tau \in \mathcal{T}} h_{\tau}
$$


Our analysis uses the condition that the mesh $\mathcal{T}$ is globally quasi-uniform, i.e. $h \approx h_{\tau}$ for all $\tau \in \mathcal{T}$. We stress again, there is no assumption that along an interface $\gamma_{i j}$ the triangulations $\mathcal{T}_{i}$ and $\mathcal{T}_{j}$ produce the same mesh.

Let $V_{i, h}$ be the conforming (see, ([12]) finite element space of piece-wise linear functions associated with the triangulation $\mathcal{T}_{i}$. Further, let $V_{h}:\left.V_{h}\right|_{\Omega_{i}}=V_{i, h}$, for $i=1, \ldots, p$, be the finite element space on $\mathcal{T}$. The functions in $V_{h}$ are, in general, discontinuous across $\gamma_{i j}$. However, their traces on $\gamma_{i j}$ from $\Omega_{i}$ and $\Omega_{j}$ are welldefined.

Let $I_{h}: V \rightarrow V_{h}$ be an operator such that for $u \in H^{2-\beta}(\Omega)$ and $0 \leq \beta \leq 1$ :

$$
\begin{aligned}
h^{-1}\left\|u-I_{h} u\right\|_{L^{2}(\Omega)}+\left\|u-I_{h} u\right\|_{H^{1}(\Omega)} & +h^{-1 / 2}\left\|u-I_{h} u\right\|_{L^{2}(\Gamma)} \\
& \preceq h^{1-\beta}\|u\|_{H^{2-\beta}(\Omega)} .
\end{aligned}
$$

Now the interior penalty finite element method reads as: Find $u_{h}^{\epsilon} \in V_{h}$ such that

$$
A^{\epsilon}\left(u_{h}^{\epsilon}, \phi\right):=a\left(u_{h}^{\epsilon}, \phi\right)+\epsilon^{-1} c\left(\Lambda u_{h}^{\epsilon}, \Lambda \phi\right)=f(\phi) \quad \text { for all } \phi \in V_{h} .
$$

Obviously, the bilinear form $A^{\epsilon}(\cdot, \cdot)$ is symmetric and positive definite on $V_{h} \times$ $V_{h}$. Therefore, the corresponding finite element "stiffness" matrix is symmetric and positive definite and the finite element system has am unique solution.

Now we derive an error estimate for the finite element interior penalty method. According to our construction $V=\sum H^{1}\left(\Omega_{i}\right) \cap H_{0}^{1}(\Omega)$ and

$$
(w, v)_{V}=\sum_{i} \int_{\Omega_{i}}(\nabla w \cdot \nabla v+w v) d x .
$$

Since the number of subdomains $p$ is finite and all $\Omega_{i}$ are shape-regular, it follows that $A^{1}(v, v)$ is uniformly equivalent to the norm $\|v\|_{V}^{2}$ and the inequality (4.1) holds. Therefore, the results of the previous sections are valid and we can apply Theorem 5.1.

The error estimate is almost immediate consequence of Theorem 5.1 and the approximation property (6.1) of the space $V_{h}$. Indeed, the error $u_{\epsilon}-u_{h}^{\epsilon}$ satisfies the orthogonality property

$$
A_{h}^{\epsilon}\left(u_{\epsilon}-u_{h}^{\epsilon}, \phi\right)=0 \text { for all } \phi \in V_{h} .
$$

Using the coercivity of $A^{\epsilon}(\cdot, \cdot)$, we get

$$
\begin{aligned}
\left\|u_{\epsilon}-u_{h}^{\epsilon}\right\|_{V}^{2} & \preceq A^{\epsilon}\left(u_{\epsilon}-u_{h}^{\epsilon}, u_{\epsilon}-u_{h}^{\epsilon}\right) \\
& \preceq \inf _{v \in V_{h}} A^{\epsilon}\left(u_{\epsilon}-v, u_{\epsilon}-v\right) \\
& \preceq A^{\epsilon}\left(u_{\epsilon}-u, u_{\epsilon}-u\right)+A^{\epsilon}\left(u-I_{h} u, u-I_{h} u\right) .
\end{aligned}
$$

Now the estimates (5.3) and (6.1) produce the following result:

$$
\begin{aligned}
\left\|u-u_{h}^{\epsilon}\right\|_{V} & \preceq\left\|u-u_{\epsilon}\right\|_{V}+\left\|u_{\epsilon}-u_{h}^{\epsilon}\right\|_{V} \\
& \preceq\left(\epsilon^{1-\delta}|\log \epsilon|^{1-2 \delta}+h^{1-\delta}+\epsilon^{-1 / 2} h^{3 / 2-\delta}\right)\|u\|_{H^{2-\delta}(\Omega)}
\end{aligned}
$$

for $u \in H^{2-\delta}(\Omega), 0 \leq \delta<1 / 2$. 
The above estimates suggest that for the penalty parameter $\epsilon \approx h$ we get almost optimal convergence rate. This result is stated in the following theorem:

Theorem 6.1. Assume that the solution $u$ of the problem (2.1) belongs to $H^{2-\delta}(\Omega)$ for some $0 \leq \delta<1 / 2$. Then the solution $u_{h} \in V_{h}$ of the interior penalty finite element method

$$
a_{h}\left(u_{h}, \phi\right)+h^{-1} c\left(\Lambda u_{h}, \Lambda \phi\right)=f(\phi) \quad \text { for all } \phi \in V_{h}
$$

exists and satisfies the apriori error estimate

$$
\left\|u-u_{h}\right\|_{V} \preceq h^{1-\delta}|\log h|^{1-2 \delta}\|u\|_{H^{2-\delta}(\Omega)} .
$$

Moreover, the condition number of the corresponding finite element "stiffness" matrix is the same as in the case of standard Galerkin method with linear elements, namely, $O\left(h^{-2}\right)$.

6.2. Numerical tests. The performance of the proposed penalty method is tested on two model examples for the Poisson equation on the unit square with Dirichlet boundary conditions. Our finite element implementation handles arbitrary triangulations of the domain and linear finite elements.

In the table below we present the error $u-u_{h}$ measured in discrete $L^{2}$ and $H^{1}$ norms for two test problems for the Poisson equation. The domain is split into four equal subdomains that are triangulated independently so that the meshes do not match along the interface $\Gamma$. The test problems are designed to check the accuracy of the interior penalty method. The first example has exact solution $u\left(x_{1}, x_{2}\right)=$ $\sin ^{2}\left(2 \pi x_{1}\right) \sin ^{2}\left(2 \pi x_{2}\right)$ so that the normal derivative along the interfaces $\gamma_{i j}$ is zero. This means that the interior penalty method should have the same accuracy as the standard Galerkin method in both $L^{2}$-and $H^{1}$-norms. This is readily observed from Table 1 . The second test problem has exact solution $u\left(x_{1}, x_{2}\right)=x_{1}^{2}+x_{2}^{2}$. We have observed from our computations that the interface is the main contributor to the error. Note that the convergence in $L^{2}$-norm is of first order, while the convergence in $H^{1}$-norm is approximately first order. In the discrete $L^{2}$ and $H^{1}$-norms the relative

\begin{tabular}{|c|r|r|r|r|r|r|}
\hline \multicolumn{2}{|c|}{} & \multicolumn{2}{|c|}{$\begin{array}{c}\text { exact solution } u \\
\sin ^{2}\left(2 \pi x_{1}\right) \sin ^{2}\left(2 \pi x_{2}\right)\end{array}$} & \multicolumn{2}{c|}{$\begin{array}{c}\text { exact solution } u \\
x_{1}^{2}+x_{2}^{2}\end{array}$} \\
\hline level & \# nodes & $L^{2}$-error & $H^{1}$-error & $L^{2}$-error & $H^{1}$-error & cond. \# \\
\hline 1 & 65 & 0.055362 & 1.094882 & 0.004712 & 0.066380 & 30 \\
\hline 2 & 225 & 0.015679 & 0.589709 & 0.002957 & 0.037646 & 105 \\
\hline 3 & 833 & 0.004017 & 0.300594 & 0.001056 & 0.020850 & 439 \\
\hline 4 & 3201 & 0.001011 & 0.151084 & 0.000545 & 0.011459 & 1829 \\
\hline 5 & 12545 & 0.000253 & 0.075646 & 0.000285 & 0.006398 & 7385 \\
\hline 6 & 52695 & 0.000063 & 0.037836 & 0.000141 & 0.003535 & 29438 \\
\hline order & & $\approx 2$ & $\approx 1$ & $\approx 1$ & $\approx 0.91$ & \\
\hline \hline
\end{tabular}

TABLE 1. Numerical results for four subdomains with non-matching grids 
error on the finest (6th) level is $0.03 \%$ and $1.95 \%$ for the exact solution $u\left(x_{1}, x_{2}\right)=$ $\sin ^{2}\left(2 \pi x_{1}\right) \sin ^{2}\left(2 \pi x_{2}\right)$ and $0.08 \%$ and $0.72 \%$ for the exact solution $u\left(x_{1}, x_{2}\right)=x_{1}^{2}+x_{2}^{2}$.

Additional numerical examples are reported in [20], including condition number estimates and accuracy results for various test problems.

Acknowledgment. Part of this research has been done during the summer visits of the first and the second authors to Lawrence Livermore National Laboratory. The authors thank the Institute for Scientific Computing Research and the Center for Applied Scientific Computing for their hospitality and for the technical and financial support.

\section{REFERENCES}

[1] T. Arbogast, L. C. Cowsar, M. F. Wheeler, and I. Yotov, Mixed finite element methods on non-matching multi-block grids, SIAM J. Numer. Anal., 37 (2000) 1295-1315.

[2] D. Arnold, An interior penalty finite element method with discontinuous elements, SIAM J. Numer. Anal. 19 (1982) 742-760.

[3] D. Arnold, F. Brezzi, B. Cockburn, and L.D. Marini, Unified analysis of discontinuous Galerkin methods for elliptic problems, SIAM J. Numer. Anal. (submitted).

[4] I. Babuška, The finite element method for elliptic equations with discontinuous solutions, Computing, 5 (1970), 207-213.

[5] P.E. Bjorstad, M.S. Espedal, and D.E. Keyes, Eds., 9th International Conference on Domain Decomposition Methods, Ullensvang, Norway, 1996, Published by ddm.org, 1998.

[6] J.H. Bramble, J.E. Pasciak, and A. Schatz, The construction of preconditioners for elliptic problems by substructuring. I, Math. Comp., 47 (1986), 103-134.

[7] J.H. Bramble, J.E. Pasciak, and A. Schatz, The construction of preconditioners for elliptic problems by substructuring. IV, Math. Comp., 53 (1989), 1-24.

[8] J.H. Bramble, J. E. Pasciak, and P. S. Vassilevski, Computational scales of Sobolev norms with application to preconditioning, Math. Comp., 69 (2000) 463-480.

[9] F. Brezzi and M. Fortin, Mixed and Hybrid Finite Element Methods, Springer, Berlin, Heidelberg, New York, 1991

[10] Y. Cao and M.D. Gunzburger, Least-squares finite element approximations to solutions of interface problems, STAM J. Numer. Anal., 35 (1998) 393-405.

[11] T.F. Chan, T. Kako, H. Kawarada, and O. Pironneau, Eds., Domain Decomposition Methods in Science and Engineering, 12-th International Conference in Chiba, Japan, 1999, Published by ddm.org, 2001 .

[12] P.G. Ciarlet, The finite element method for elliptic problems, North Holland, Amsterdam (1978).

[13] B. Cockburn and C. Dawson, Approximation of the velocity by coupling discontinuous Galerkin and mixed finite element methods for flow problems, Preprint, (2001).

[14] J. Douglas and T. Dupont, Interior penalty procedures for elliptic and parabolic Galerkin methods, Lecture Notes in Physics, 58 (1978) 207-216.

[15] M. Feistauer, J. Felcman, M. Lucacova-Madvidova, and G. Warenicke, Error estimates of a combined finite volume - finite element method for nonlinear convection-diffusion problems, Preprint, (2001).

[16] P. Grisvard, Elliptic Problems in Non-smooth Domains, Pitman, Boston, 1985.

[17] C.-H. Lai, P. Bjorstad, M. Cross, amd O. Widlund, Eds., Eleventh Int. Conference on Domain Decomposition Methods, Greenwich, UK, 1998, Published by ddm.org, 1999. 
[18] R. D. Lazarov, J. E. Pasciak, and P. S. Vassilevski, Iterative solution of a coupled mixed and standard Galerkin discretization method for elliptic problems, Numer. Lin. Alg. Appl., 8 (2001) 13-31.

[19] R. D. Lazarov, J. E. Pasciak, and P. S. Vassilevski, Mixed finite element methods for elliptic problems on non-matching grids, in Large-Scale Scientific Computations of Engineering and Environmental Problems II (M. Griebel et al., Eds.), Vieweg, Notes on Numerical Fluid Mechanics, 73 (2000), 25-35.

[20] R. D. Lazarov, S. Z. Tomov, and P. S. Vassilevski, Interior penaity discontinuous approximations of elliptic problems, ISC-04-00 Technical Report, Texas A \& M University, 2000.

[21] J. L. Lions and J. Peetre, Sur une class d'espaces d'interpolation, Institute des Hautes Etudes Scientifique, Publ. Math., 19 (1994) 5-68.

[22] B. Rivière, M.F. Wheeler, and V. Girault, Improved energy estimates for the interior penalty, constrained and discontinuous Galerkin methods for elliptic problems. Part I, Computational Geosciences, 3 (1999) (3,4) 337-360.

[23] T. Rusten, P. S. Vassilevski, and R. Winther, Interior penalty preconditioners for mixed finite element approximations of elliptic problems, Math. Comp., 65 (1996), 447-466.

[24] J. Schöberl, Robust Multigrid Methods for Parameter Dependent Problems, Ph. D. Thesis, University of Linz, June 1999.

[25] C. Wieners and B. I. Wohlmuth, The coupling of mixed and conforming finite element discretizations, in Domain Decomposition Methods 10 (J. Mandel, C. Farhat, and X.-C. Cai, Eds.), Contemporary Math., \# 218 (1998), AMS, pp. 547-554.

R.D. Lazarov, Department of Mathematics, Texas A \& M Universtiy, College STATION, TX 77843, U.S.A.

E-mail address: Iazarov@math.tamu.edu

J.E. Pasciak, Department of Mathematics, Texas A \& M University, College StaTION, TX 77843, U.S.A.

E-mail address: pasciak@math.tamu.edu

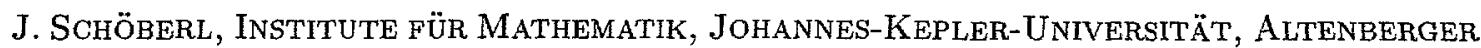

Starasse 69, A-4040 Linz, AUstria

E-mail address: joachim@math.tamu.edu

P.S. Vassilevski, Center for Applied Scientific Computing, lawrence Livermore National Laboratory, Mail Stop L-560, 7000 East Avenue, Livermore, CA 94550 , U.S.A.

E-mail address: panayot@IInl.gov 